\title{
Supramolecular Functionalized Pristine Graphene Utilizing a Bio-Compatible Stabilizer towards Ultra-Sensitive Ammonia Detection ${ }^{\dagger}$
}

\author{
Shirong Huang ${ }^{1, *}$, Luis Antonio Panes-Ruiz ${ }^{1} \oplus$, Alexander Croy ${ }^{1, *} \mathbb{C}$, Leif Riemenschneider ${ }^{1}$, \\ Vyacheslav Khavrus ${ }^{2}\left(\mathbb{D}\right.$, Viktor Bezugly ${ }^{1,2,3} \mathbb{D}$ and Gianaurelio Cuniberti ${ }^{1,3,4}(\mathbb{D})$ \\ 1 Institute for Materials Science and Max Bergmann Center for Biomaterials, Technische Universität Dresden, \\ 01062 Dresden, Germany; antonio.panes@gmail.com (L.A.P.-R.); Leif.Riemenschneider@tu-dresden.de (L.R.); \\ viktor.bezugly@tu-dresden.de (V.B.); gianaurelio.cuniberti@tu-dresden.de (G.C.) \\ 2 Life Science Inkubator Sachsen GmbH \& Co. KG, Tatzberg 47, 01307 Dresden, Germany; \\ Khavrus@life-science-inkubator.de \\ 3 Center for Advancing Electronics Dresden (cfAED), TU Dresden, 01062 Dresden, Germany \\ 4 Dresden Center for Computational Materials Science (DCMS), TU Dresden, 01062 Dresden, Germany \\ * Correspondence: shirong.huang@tu-dresden.de (S.H.); alexander.croy@tu-dresden.de (A.C.) \\ + Presented at the 8th International Symposium on Sensor Science, 17-28 May 2021; Available online: \\ https://i3s2021dresden.sciforum.net/.
}

check for

updates

Citation: Huang, S.; Panes-Ruiz, L.A.; Croy, A.; Riemenschneider, L.; Khavrus, V.; Bezugly, V.; Cuniberti, G. Supramolecular Functionalized Pristine Graphene Utilizing a BioCompatible Stabilizer towards UltraSensitive Ammonia Detection. Eng. Proc. 2021, 6, 14. https://doi.org/ 10.3390/I3S2021Dresden-10089

Academic Editors: Gianaurelio Cuniberti and Larysa Baraban

Published: 17 May 2021

Publisher's Note: MDPI stays neutral with regard to jurisdictional claims in published maps and institutional affiliations.

Copyright: (C) 2021 by the authors. Licensee MDPI, Basel, Switzerland. This article is an open access article distributed under the terms and conditions of the Creative Commons Attribution (CC BY) license (https:// creativecommons.org/licenses/by/ $4.0 /)$.

\begin{abstract}
Recently, graphene has attracted intensive attention in the gas sensing field due to its high electrical conductivity as well as large specific surface areas. Lots of graphene-based gas sensors have been reported with excellent gas sensing performance. However, the sensing element materials for most of the above sensors actually consist of a reduced graphene oxide (rGO) derivative rather than pristine graphene, such as $\mathrm{rGO}, \mathrm{rGO} /$ metal particle, $\mathrm{rGO}$ /polymers, etc. Complex chemical oxidation and reduction are usually involved for the preparation of reduced graphene oxide derivatives. Even though there are some pristine graphene-based gas sensors synthesizing with the approaches of chemical vapor deposition (CVD) or mechanical cleavage, the high cost of the set-up or the low productivity cannot decrease the cost of the practical sensors. In this work, we develop pristine graphene-based gas sensors utilizing flavin monocleotide sodium salt (FMNS) toward ultra-sensitive ammonia detection. The sensor has 3\% response upon exposure to $10 \mathrm{ppm}$ $\mathrm{NH}_{3}$ and a limit of detection of $1.6 \mathrm{ppm}$ at room temperature and shows a good recovery. Raman, UV-Vis, FT-IR spectra, as well as scanning electron microscope (SEM) measurements are employed to characterize the quality of the graphene flakes, indicating a good structural quality of graphene with few defects. The effects of the concentration of graphene dispersion functionalized by FMNS on the sensing performance towards ammonia sensing were also investigated. The process is very mild, environmentally friendly, and low cost. We believe this work may pave a path to design a high-performance gas sensor with low cost and boost the application of graphene for sensing.
\end{abstract}

Keywords: graphene-based sensor; flavin monocleotide sodium salt; ammonia detection

Institutional Review Board Statement: Not applicable.

Informed Consent Statement: Not applicable.

Data Availability Statement: https://www.mdpi.com/ethics. 